\title{
Tolerance of bivalve mollusc hemocytes to variable oxygen availability: a mitochondrial origin?
}

\author{
Ludovic DonaghY a , Sébastien ArTigaud, Rossana Sussarellu, Christophe LAMBERT, Nelly Le GoÏc, \\ Hélène HÉGARET and Philippe SOUDANT ${ }^{\mathrm{b}}$ \\ Université de Bretagne Occidentale, IUEM, LEMAR Laboratoire des Sciences de l’Environnement marin, UMR 6539, 29280 Plouzané, France
}

Received 24 January 2013; Accepted 6 June 2013

\begin{abstract}
Bivalve molluscs survive lack of oxygen and may present cellular characteristics allowing tolerance to oxygen deprivation. The objective of the present work was to determine in vitro responses of circulating cells (i.e., hemocytes) of Pacific oyster, Crassostrea gigas, to oxygen deprivation by analysis of mitochondrial membrane potential $(\Delta \Psi \mathrm{m})$ and reactive oxygen species (ROS) production. Hemocytes submitted to oxygen deprivation showed a reduced ROS production and an increased $\Delta \Psi \mathrm{m}$. Confrontation of present results with recently reported findings allows us to hypothesize the existence of an alternative mitochondrial reductase, and the involvement of mitochondrial potassium channel in maintenance of $\Delta \Psi \mathrm{m}$. Tolerance of bivalve hemocytes to variable oxygen availability appears, at least partially, related to mitochondrial specificities.
\end{abstract}

Keywords: Hemocyte / Hypoxia / Mitochondria / Reactive oxygen species / Flow cytometry / Oyster

\section{Introduction}

Intertidal bivalve molluscs are highly tolerant to variable oxygen $\left(\mathrm{O}_{2}\right)$ availability, surviving complete lack of $\mathrm{O}_{2}$ for prolonged periods. For instance, they are naturally submitted to $\mathrm{O}_{2}$ deprivation twice-daily during low tide. In the whole animal kingdom, it appears that the key adaptation to $\mathrm{O}_{2}$ deprivation in highly tolerant species is a simultaneous reduction in metabolic rate and metabolic demands (Bickler and Buck 2007). Mitochondria are organelles that generate most of the cell's supply of energy during respiration. Cellular respiration is characterized by the reduction of $\mathrm{O}_{2}$ into water $\left(\mathrm{H}_{2} \mathrm{O}\right)$, and the transport of electrons all along molecular complexes located on the inner mitochondrial membrane. During electron transport, protons are also transferred across the inner mitochondrial membrane, producing an electrochemical gradient composed of both a mitochondrial membrane potential $(\Delta \Psi \mathrm{m})$ and a $\mathrm{pH}$ gradient. Part of consumed $\mathrm{O}_{2}$ is not completely reduced to water but instead partially reduced into reactive oxygen species (ROS). It has been hypothesized a dependence of ROS production on available $\mathrm{O}_{2}$ (Turrens 2003).

\footnotetext{
${ }^{a}$ Current address: Florida Gulf Coast University, Dep. Marine and Ecological Sciences, College of Arts and Sciences, 10501 FGCU Boulevard, Fort Myers, 33965-6565, USA

b Corresponding author: philippe.soudant@univ-brest.fr
}

Hemocytes are a major component of bivalve homeostasis through their involvement in physiological mechanisms such as internal defense, tissue and shell repair, nutrient digestion and transportation, as well as detoxification of biotic and abiotic pollutants (Donaghy et al. 2009). Two main hemocyte populations are generally described, granulocytes and hyalinocytes, based on the microscopically-determined presence or absence of intracytoplasmic granules (Donaghy et al. 2009). Hemocytes are freely circulating in hemolymph, a semi-open circulatory system whose physico-chemical characteristics follow variations of external environment, at least as long as shell valves remain open (Boyd and Burnett 1999). Hemocytes might then be directly exposed to environmental modifications, including $\mathrm{O}_{2}$ deprivation.

Bivalve molluscs have developed capabilities to survive $\mathrm{O}_{2}$ deprivation far better than can other animals on average. Until now, only very few studies investigated the cellular responses of bivalves to oxygen deprivation (Mosca et al. 2013). We very recently characterized the involvement of mitochondria in intracellular ROS production in hemocytes of the Pacific oyster Crassostrea gigas and pointed out some differences with mammalian models (Donaghy et al. 2012). In light of this new knowledge, the objective of the present work was to determine if response of hemocytes to low $\mathrm{O}_{2}$ availability could be related to mitochondrial specificities. Viability, intracellular oxidative 
activity and $\Delta \Psi \mathrm{m}$ were flow cytometrically analyzed in hemocytes submitted to $\mathrm{O}_{2}$ deprivation in vitro.

\section{Materials and methods}

\subsection{Animals}

Adult Pacific oysters, Crassostrea gigas, were collected on natural beds from the Bay of Brest and placed in $500 \mathrm{~L}$ flowthrough seawater tanks (salinity 34 ; temperature $13{ }^{\circ} \mathrm{C}$ ) and held for one week prior experiments.

\subsection{Hemolymph and experimental design}

Using a syringe fitted with a $22-\mathrm{G}$ needle, 1.5 to $2.0 \mathrm{ml}$ of hemolymph were withdrawn from the adductor muscle of each oyster through a notch ground extemporaneously. Collected hemolymph was then immediately transferred into micro-tubes maintained on ice, to minimize cell clumping. Hemolymph from individuals was examined microscopically for purity and then filtered through an $80 \mu \mathrm{m}$ nylon mesh. All subsequent analyses were performed on individual samples.

Ten parafilm-sealed vials containing $2.4 \mathrm{ml}$ of filtered sterile seawater (FSSW) were placed under a nitrogen evaporator (Nevap-12, Organomation Associates, Berlin, MA, USA; 0.05 bar). First, evaporator needles were placed above the solution during $30 \mathrm{~min}$ for nitrogen saturation of gas phase, and then sank into FSSW for oxygen removal. After $1 \mathrm{~h}$, oxygen concentration in seawater was reduced by $85 \%$, needles were then removed and vials were maintained sealed.

Hemolymph from 10 oysters was then withdrawn as described above and $600 \mu \mathrm{l}$ from each individual were introduced into the previously prepared vials and placed on ice to reduce cell aggregation. In parallel, $600 \mu \mathrm{l}$ from the same individuals were introduced into vials containing $2.4 \mathrm{ml}$ of control FSSW (i.e., which has not been placed on nitrogen evaporator).

After $90 \mathrm{~min}, 400 \mu \mathrm{l}$ from each condition were then transferred into flow cytometry tubes to determine cellular parameters.

\subsection{Flow cytometry}

Cellular parameters were evaluated using flow cytometer FACScalibur (Becton-Dickinson) equipped with argon laser $488 \mathrm{~nm}$. Collected data were analyzed using WinMDI 2.9 software. After incubation of cells with fluorescent probes, samples were placed and kept on ice until flow cytometric data acquisition.

\subsubsection{Hemocyte morphology and mortality}

Morphology and mortality of hemocytes were evaluated using a double staining procedure including SYBR Green I (Invitrogen) and propidium iodide (PI, Sigma).

Morphology: SYBR Green I is a membrane-permeable fluorescent dye that binds to double-stranded DNA and then emits green fluorescence $(520 \mathrm{~nm})$. Detection of this fluorescence allows distinction between single cells and aggregates as well as debris. Hemocyte morphology was based upon relative flow-cytometric parameters, forward scatter (FSC, particle size) and side scatter (SSC, internal complexity); and allowed distinction between hyalinocytes and granulocytes. Morphological parameters are expressed in arbitrary flow-cytometric units (A.U.).

Mortality: membranes of viable cells do not allow PI to penetrate; whereas, altered membranes are permeable to PI. Dead cells are characterized by loss of membrane integrity and are, therefore, double stained by SYBR Green I and PI. Hemocyte mortality is expressed as the percentage of SYBR Green and PI positive cells.

Briefly, SYBR Green I (1/1000 of stock solution) and PI (20 $\mu \mathrm{g} \mathrm{ml}^{-1}$ ) were added to previously prepared flow cytometry tubes (Sect. 2.2). Tubes were maintained sealed and incubation was performed for $120 \mathrm{~min}$ in the dark at $10^{\circ} \mathrm{C}$, prior to flow cytometric data acquisition.

\subsubsection{Hemocyte oxidative activity}

Determination of oxidative activity was performed using 2'7'-dichlorofluorescein diacetate (DCFH-DA; Molecular Probes, Invitrogen, Carlsbad, CA, USA), a membrane permeable, non-fluorescent probe. Inside hemocytes, the -DA radical is first hydrolyzed by esterase enzymes. Intracellular hydrogen peroxide $\left(\mathrm{H}_{2} \mathrm{O}_{2}\right)$, as well as superoxide ion $\left(\mathrm{O}_{2}^{-}\right)$, then oxidizes DCFH to the fluorescent DCF molecule. Oxidation of DCFH can also be mediated by various oxidase and peroxidase enzymes. DCF green fluorescence, detected on the FL1 detector of the flow cytometer, is proportional to the total oxidative activity of hemocytes, including reactive oxygen species (ROS) production.

Briefly, DCFH-DA (10 $\mu \mathrm{M})$ was added to previously prepared flow cytometry tubes (Sect. 2.2). Tubes were maintained sealed and incubation was performed for 120 min in the dark at $10{ }^{\circ} \mathrm{C}$, prior to flow cytometric data acquisition. Relative oxidative activity is expressed as the level of green fluorescence (FL1 detector of the flow cytometer) in arbitrary units (A.U.).

\subsubsection{Mitochondrial membrane potential}

Estimation of mitochondrial membrane potential $(\Delta \Psi \mathrm{m})$ was performed using JC-10 (Interchim, Montluçon, France), a membrane permeable fluorescent probe. JC-10 accumulates and aggregates in mitochondria, selectively generating an orange emission profile $(590 \mathrm{~nm})$. As membrane potential decreases, JC-10 monomers are generated, resulting in a shift to green fluorescence emission $(525 \mathrm{~nm})$. The ratio between both fluorescence intensities can then be calculated to provide information about $\Delta \Psi \mathrm{m}$.

Thus, JC-10 $(10 \mu \mathrm{M})$ was added to previously prepared flow cytometry tubes. Tubes were maintained sealed and incubation was performed for $30 \mathrm{~min}$ in the dark at $10^{\circ} \mathrm{C}$, prior to flow cytometric data acquisition. Relative $\Delta \Psi \mathrm{m}$ intensity is expressed as the ratio between the levels of orange fluorescence (FL2 detector of the flow cytometer) and green fluorescence (FL1 detector). 


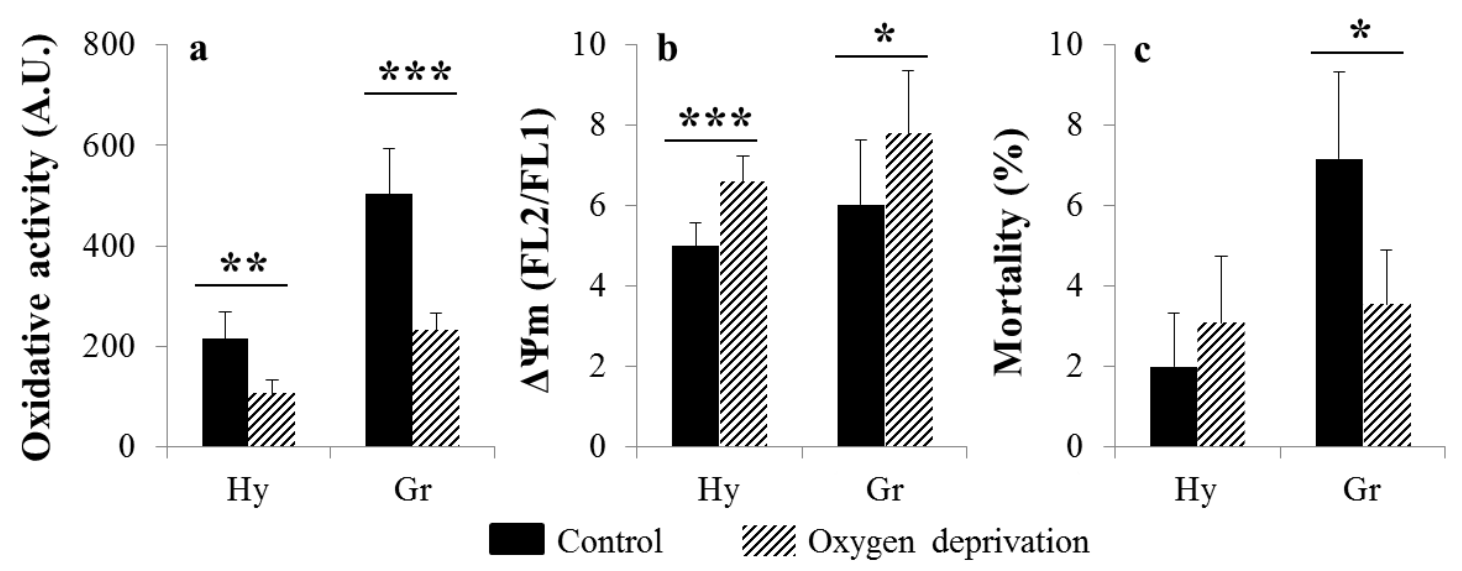

Fig. 1. Intracellular oxidative activity (a), mitochondrial membrane potential, $\Delta \Psi \mathrm{m}$ (b), and mortality (c) of hemocytes submitted to deprivation of oxygen. Hyalinocytes (Hy), Granulocytes (Gr). A.U.: arbitrary units. FL ratio: orange/green fluorescence levels. Values are reported as mean $\pm 95 \% \mathrm{CI}(N=10)$. Asterisks indicate a significant difference between control and oxygen deprivation conditions, $* p \leqslant 0.05, * * p \leqslant 0.01$, $* * * p \leqslant 0.001$ (paired $t$-test or Mann-Whitney).

\subsection{Statistical analyses}

Prior statistical analysis, percentages were transformed as Arcsin of the square root. Similarity of variances was assessed using F-test. If variances were similar, paired $t$-test was performed to compare hemocyte parameters under $\mathrm{O}_{2}$ deprivation against control condition. If data did not follow normal distribution, Mann-Whitney test was performed. Statistical analysis was performed using Statgraphics Plus 5.1 software (Manugistics, Inc., Rockville, MD, USA). Results are displayed as mean $\pm 95 \%$ confidence interval (CI). Differences were considered significant at $p \leqslant 0.05$.

\section{Results and discussion}

When the Pacific oyster Crassostrea gigas is exposed to air, shell closure occurs instantaneously, effectively isolating the animal from ambient environment. One consequence of this behavior is the cessation of gas exchange between the oyster and the external medium (Boyd and Burnett 1999). Oxygen concentration $\left(\left[\mathrm{O}_{2}\right]\right)$ inside the pallial cavity then decreases very quickly to reach undetectable concentration within minutes (data not shown). Due to the anatomy of oyster's circulatory system, physico-chemical characteristics of hemolymph readily follow pallial fluid variables, as reported by Boyd and Burnett (1999) in the eastern oyster Crassostrea virginica submitted to low levels of available $\mathrm{O}_{2}$. Hemocytes circulating in hemolymph might then be rapidly impacted by $\mathrm{O}_{2}$ deprivation.

When hemocytes were submitted to $\mathrm{O}_{2}$ deprivation, intracellular oxidative activity in hyalinocytes and granulocytes was significantly reduced from $215 \pm 52$ to $106 \pm 27$ A.U. and from $504 \pm 89$ to $232 \pm 32$ A.U., respectively (Fig. 1a), as previously reported for Crassostrea virginica (Boyd and Burnett 1999). Donaghy et al. (2012) showed that reactive oxygen species (ROS) account for the main part of intracellular oxidative activity in hemocytes, and originate from mitochondrial respiratory complexes. It is currently recognized that mitochondrial complexes are organized into supramolecular assemblies called supercomplexes or respirasomes (Genova and Lenaz 2013). There are some indications that, in conditions such as reduced $\mathrm{O}_{2}$ availability, supercomplex association may modify the conformation of partner complexes in such a way as to enhance their catalytic activity (Genova and Lenaz 2013). Indeed, Ramírez-Aguilar et al. (2011) reported, in plant mitochondrial supercomplexes, an increased activity of complex IV with low $\left[\mathrm{O}_{2}\right]$. Similarly, Sussarellu et al. (2013) suggested an increased capacity of mitochondrial complex IV in gills of Pacific oyster submitted to reduced $\mathrm{O}_{2}$ concentration. In these conditions, complex IV may display higher affinity for electrons, reducing the potential for production of reactive oxygen species (ROS) (Fig. 2).

In mammalian models, cells challenged with low $\left[\mathrm{O}_{2}\right]$ contrastingly show an overproduction of ROS (Solaini et al. 2010). In mammals, the major sites of ROS production are within mitochondrial complexes I and III, being prevalent the contribution of complex I (Solaini et al. 2010). When mammalian cells are submitted to low $\left[\mathrm{O}_{2}\right]$, increased ROS production is due primarily to reverse electron transport from complex III to complex I (Bickler and Buck 2007). Interestingly, in hemocytes, complex I does not appear as a major site of ROS production (Donaghy et al. 2012). Furthermore, we recently showed that, while inhibition of respiratory chain between complex III and cytochrome $\mathrm{C}$ by antimycin A in mammalian cells results in a ROS overproduction, such inhibition results in a decrease of ROS production in hemocytes of $C$. $g i$ gas (Donaghy et al. 2012). In hemocytes, when complex III is inhibited, either with antimycin $\mathrm{A}$ or during $\mathrm{O}_{2}$ deprivation, ROS formation may be avoided by shunting electrons via alternative pathway(s) (Fig. 2). Although Abele et al. (2007) suggested the involvement of the alternative oxidase (AOX), this is unlikely to happen since AOX expression and activity in bivalve increased during re-oxygenation phases (Sussarellu et al. 2012 ; 2013) as well as in context of highly oxygenated environments (Abele et al. 2007). Furthermore, simultaneous inhibition of AOX and complex III did not result in an increased ROS production (Donaghy et al. 2012). It can therefore be hypothesized the existence of an alternative terminal reductase 


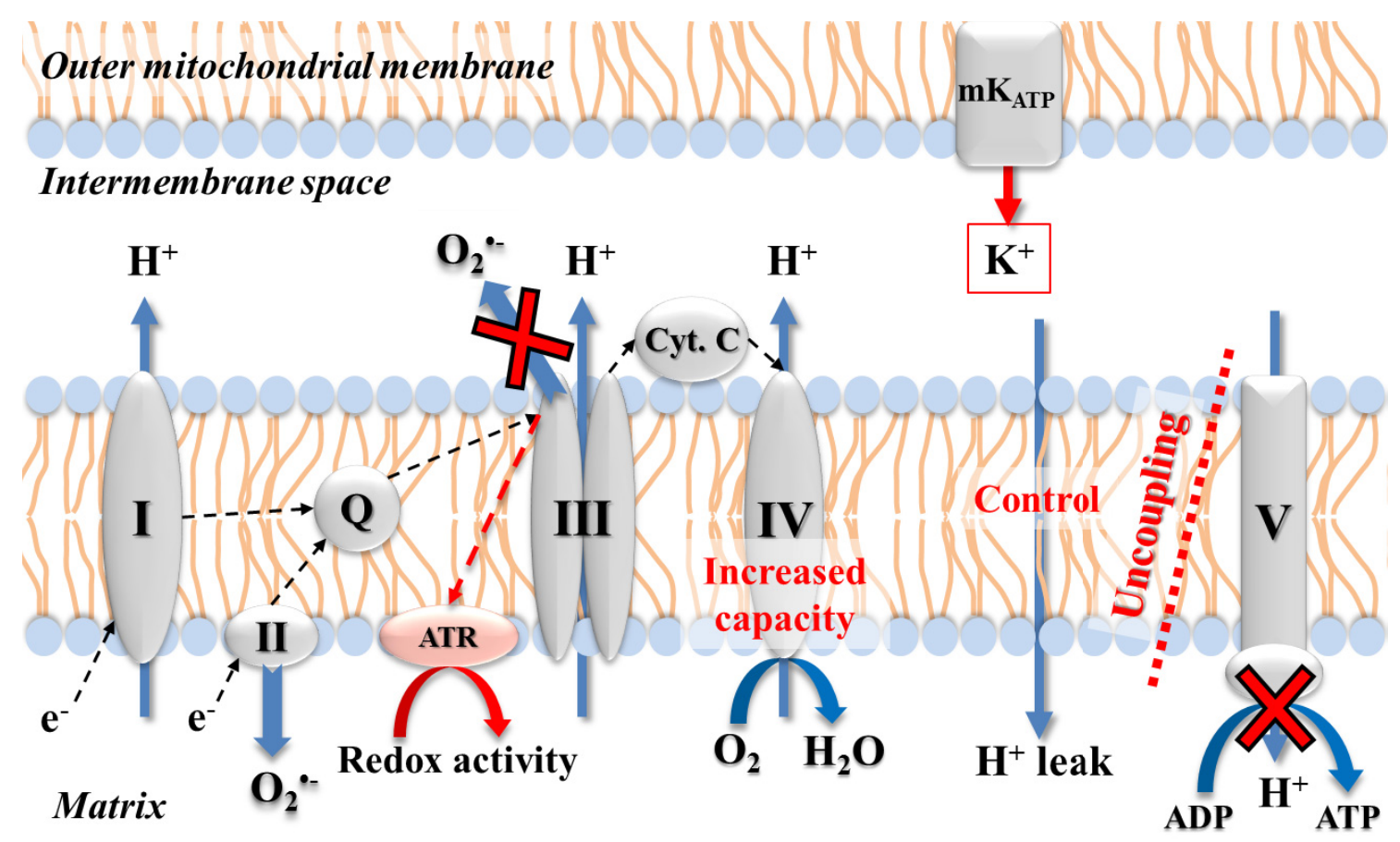

Fig. 2. Hypothetical overview of bivalve hemocytes' mitochondrial electron and proton flux under low oxygen availability. (1) When oxygen is available, electrons $\left(\mathrm{e}^{-}\right)$released from reduced cofactors flow from mitochondrial complexes I and II, to complexes III and IV (black dotted line) through coenzyme $\mathrm{Q}(\mathrm{Q})$ and cytochrome $\mathrm{C}\left(\mathrm{Cyt}\right.$. C). A proton $\left(\mathrm{H}^{+}\right)$flux from mitochondrial matrix to intermembrane space (blue arrows) is coupled with electron transport at complexes I, III and IV. Protons then flow back to the matrix through complex V (blue arrow), driving ATP synthesis. A proton leak occurs through the membrane, from intermembrane space to mitochondrial matrix (blue arrow). Reactive oxygen species $\left(\mathrm{O}_{2}^{--}\right)$are produced at complexes II and III (Donaghy et al. 2012). (2) Under low oxygen availability, uncoupling occurs between mitochondrial electron transport chain (complexes I to IV) and complex V, which may be inhibited (red cross). ROS production may stop at complex III (red cross) and electrons may be shunted (red dotted arrow) to an unknown alternative terminal reductase (ATR) able to use substrate other than $\mathrm{O}_{2}$ as final electron acceptor. Complex IV capacity may be increased, resulting in higher affinity for electrons and lower ROS production. Mitochondrial potassium channel $\left(\mathrm{mK}_{\mathrm{ATP}}\right)$ may induce $\mathrm{K}^{+}$influx (red arrow) into mitochondrial intermembrane space to maintain an appropriate mitochondrial membrane potential $(\Delta \Psi \mathrm{m})$ in conjugation with a control of $\mathrm{H}^{+}$leak.

that would be able to use substrate other than $\mathrm{O}_{2}$ as final electron acceptor (Fig. 2). For instance, it was reported the capacity of the mussel Mytilus edulis to shunt electrons to a fumarate reductase during anaerobiosis (Tielens et al. 2002).

In the present work, mitochondrial membrane potential $(\triangle \Psi \mathrm{m})$ of hemocytes increased when submitted to $\mathrm{O}_{2}$ deprivation, from $5 \pm 0.6$ to $6.6 \pm 0.6$ in hyalinocytes and from $6 \pm 1.6$ to $7.8 \pm 1.5$ in granulocytes (Fig. 1b). In mammalian cells, decreased $\mathrm{O}_{2}$ availability contrastingly leads to a decreased $\Delta \Psi \mathrm{m}$ (Solaini et al. 2010). The reduction of proton pumping at complexes III and IV, as well as the spontaneous proton leak from intermembrane space to mitochondrial matrix lead to the reduction of $\Delta \Psi \mathrm{m}$ (Fig. 2) (Bickler and Buck 2007; Solaini et al. 2010). Maintenance of $\Delta \Psi \mathrm{m}$ is primordial, however, since its dissipation may lead to opening of mitochondrial permeability transition pore, ultimately resulting in cell death (Turrens 2003). Mammalian mitochondrial $\mathrm{F}_{0} \mathrm{~F}_{1}$-ATPase (complex $\mathrm{V}$ ) activity has been reported able to reverse, hydrolyzing ATP to transfer protons backward, from mitochondrial matrix to intermembrane space (Solaini et al. 2010). We hypothesize that species surviving long-term periods of $\mathrm{O}_{2}$ deprivation would limit ATP use to avoid threatening other vital ATP-consuming cellular processes. Consequently, complex V may likely be inhibited during low oxygen availability in bivalve; thus, increased $\Delta \Psi \mathrm{m}$ may reflect such a decrease in the proportion of phosphorylating mitochondria (Fig. 2). Sussarellu et al. (2013) suggested that, in C. gigas mitochondria, proton leak may be reduced during $\mathrm{O}_{2}$ deprivation in order to limit $\Delta \Psi \mathrm{m}$ dissipation (Fig. 2). Furthermore, activation of mitochondrial ionic channels has been reported able to protect against mitochondrial damages, ATP depletion and $\Delta \Psi \mathrm{m}$ dissipation ( $\mathrm{Xu}$ et al. 2001). For instance, activation of mitochondrial potassium channel $\left(\mathrm{mK}_{\mathrm{ATP}}\right)$ induces $\mathrm{K}^{+}$influx into the mitochondria, resulting in maintenance of $\Delta \Psi \mathrm{m}$ (Bickler and Buck 2007) (Fig. 2). Thus, hemocytes of $C$. gigas incubated with nigericin, an antiport ionophore which replaces $\mathrm{H}^{+}$by $\mathrm{K}^{+}$, similarly resulted in an increased $\Delta \Psi \mathrm{m}$ (Donaghy et al. 2012), as presently reported.

In control condition, mortality of hemocytes was low but different between both cell types: about $2 \%$ of hyalinocytes were dead while granulocytes showed $7 \%$ mortality (Fig. 1c). Such a difference in mortality between hyalinocytes and granulocytes has previously been reported in oysters (Hégaret et al. 2003) and may be related, among others, to the difference in ROS production (Fig. 1a). Indeed, ROS can induce cellular damage and are also potent cellular messengers involved in apoptotic pathways (Turrens 2003). Exposure of hemocytes to $\mathrm{O}_{2}$ deprivation resulted in no modification of 
hyalinocytes mortality but a strong decrease of granulocytes mortality (Fig. 1c), potentially linked with the concomitant decrease of ROS production (Fig. 1a). Furthermore, it has been suggested that the $\mathrm{O}_{2}$ deprivation-induced decrease of ROS production may be responsible for the activation of mitochondrial ion channels such as the $\mathrm{mK}_{\mathrm{ATP}}$ channel (Bickler and Buck 2007), as discussed above. Opening of these channels would not only help maintain $\Delta \Psi \mathrm{m}$ but also activate molecular pathways to down regulate energy-expensive cellular processes (Bickler and Buck 2007). Apoptosis of senescent or damaged cells, a natural but energy-expensive process in the turnover of hemocytes would then be limited when submitted to $\mathrm{O}_{2}$ deprivation, also leading to a decrease in cell mortality (Fig. 1c).

\section{Conclusion}

When hemocytes of the Pacific oyster were submitted to $\mathrm{O}_{2}$ deprivation, they reduced ROS production, potentially uncoupling oxidative phosphorylation and mitochondrial electron transport chain. Hemocytes also displayed an increased $\Delta \Psi \mathrm{m}$ which may reflect the decrease in the proportion of phosphorylating mitochondria. These cellular responses differ from mammalian models and correlate with variances previously reported in hemocytes' mitochondrial metabolism (Donaghy et al. 2012). Tolerance of bivalve hemocytes to variable $\mathrm{O}_{2}$ availability then appears, at least partially, dependent on mitochondrial specificities. Present results warrant further investigation for better comprehension of cellular adaptations to variable oxygen availability.

Acknowledgements. The present work has been supported by a postdoctoral grant from CNRS INEE to L. Donaghy.

\section{References}

Abele E., Philip E., Gonzalez P.M., Puntarulo S., 2007, Marine invertebrate mitochondria and oxidative stress. Front. Biosci. 12, 933-946.

Bickler P.E., Buck L.T., 2007, Hypoxia tolerance in reptiles, amphibians, and fishes: life with variable oxygen availability. Annu. Rev. Physiol. 69, 145-170.
Boyd J.N., Burnett L.E., 1999, Reactive oxygen intermediate production by oyster hemocytes exposed to hypoxia. J. Exp. Biol. 202, 3135-3143.

Donaghy L., Kraffe E., Le Goïc N., Lambert C., Volety A.K., Soudant P., 2012, Reactive oxygen species in unstimulated hemocytes of the Pacific oyster Crassostrea gigas: a mitochondrial involvement. PLoS One 7, e46594.

Donaghy L., Lambert C., Choi K.-S., Soudant P., 2009, Hemocytes of the carpet shell clam (Ruditapes decussatus) and the Manila clam (Ruditapes philippinarum): current knowledge and future prospects. Aquaculture 297, 10-24.

Genova M.L., Lenaz G., 2013, A critical appraisal of the role of respiratory supercomplexes in mitochondria. Biol. Chem. 394, 631-639.

Hégaret H., Wikfors G., Soudant P., 2003, Flow cytometric analysis of haemocytes from eastern oysters, Crassostrea virginica, subjected to a sudden temperature elevation II. Haemocyte functions: aggregation, viability, phagocytosis, and respiratory burst. J. Exp. Mar. Biol. Ecol. 293, 249-265.

Mosca F., Narcisi V., Calzetta A., Gioia L., Finoia M.G., Latini M., Tiscar P.G., 2013, Effects of high temperature and exposure to air on mussel (Mytilus galloprovincialis Lmk 1891) hemocyte phagocytosis: modulation of spreading and oxidative response. Tissue Cell 45, 198-203.

Ramírez-Aguilar S.J., Keuthe M., Rocha M., Fedyaev V.V., Kramp K., Gupta K.J., Rasmusson A.G., Schulze W.X., van Dongen J.T., 2011, The composition of plant mitochondrial supercomplexes changes with oxygen availability. J. Biol. Chem. 286, 43045-43053.

Solaini G., Baracca A., Lenaz G., Sgarbi G., 2010, Hypoxia and mitochondrial oxidative metabolism. Biochim. Biophys. Acta Bioenerg. 1797, 1171-1177.

Sussarellu R., Fabioux C., Camacho Sanchez M., Le Goïc N., Lambert C., Soudant P., Moraga D., 2012, Molecular and cellular response to short-term oxygen variations in the Pacific oyster Crassostrea gigas. J. Exp. Mar. Biol. Ecol. 412, 87-95.

Sussarellu R., Dudognon T., Fabioux C., Soudant P., Moraga D., Kraffe E., 2013, Rapid mitochondrial adjustments in response to short-term hypoxia and re-oxygenation in the Pacific oyster Crassostrea gigas. J. Exp. Biol. 216, 1561-1569.

Tielens A.G.M., Rotte C., van Hellemond J.J., Martin W., 2002, Mitochondria as we don't know them. Trends Biochem. Sci. 27, 564-572.

Turrens J.F., 2003, Mitochondrial formation of reactive oxygen species. J. Physiol. 552, 335-344.

Xu M., Wang Y., Ayub A., Ashraf M., 2001, Mitochondrial K-ATP channel activation reduces anoxic injury by restoring mitochondrial membrane potential. Am. J. Physiol. Heart Circ. Physiol. 281, H1295-H1303. 